\title{
Review
}

Cerebrovascular Diseases
Cerebrovasc Dis 2013;35:430-443

DOI: $10.1159 / 000350199$
Received: June 7, 2012

Accepted: February 14, 2013

Published online: May 31, 2013

\section{Stroke-Associated Pneumonia: Major Advances and Obstacles}

\author{
Yousef Hannawi $^{\mathrm{a}}$ Bashar Hannawi ${ }^{\mathrm{b}}$ Chethan P. Venkatasubba Rao ${ }^{\mathrm{a}}$ \\ Jose I. Suarez ${ }^{\mathrm{a}}$ Eric M. Bershad ${ }^{\mathrm{a}}$ \\ ${ }^{a}$ Department of Neurology, Baylor College of Medicine, and ${ }^{b}$ Department of Medicine, \\ The Methodist Hospital, Houston, Tex., USA
}

\section{Key Words}

Acute ischemic stroke · Post-stroke pneumonia .

Stroke-induced immunodepression · Stroke-associated

pneumonia - Ventilator-associated pneumonia

\begin{abstract}
Background: Stroke-associated pneumonia (SAP) has been implicated in the morbidity, mortality and increased medical cost after acute ischemic stroke. The annual cost of SAP during hospitalization in the United States approaches USD 459 million. The incidence and prognosis of SAP among intensive care unit (ICU) patients have not been thoroughly investigated. We reviewed the pathophysiology, microbiology, incidence, risk factors, outcomes and prophylaxis of SAP with special attention to ICU studies. Methods: To determine the incidence, risk factors and prognosis of acute SAP, PubMed was searched using the terms 'pneumonia' AND 'neurology intensive unit' and the MeSH terms 'stroke' AND 'pneumonia'. Non-English literature, case reports and chronic SAP studies were excluded. Studies were classified into 5 categories according to the setting they were performed in: neurological intensive care units (NICUs), medical intensive care units (MICUs), stroke units, mixed studies combining more than one setting or when the settings were not specified and rehabilitation studies. Results: The incidences of SAP in the following settings were: NICUs 4.1$56.6 \%$, MICUs $17-50 \%$, stroke units $3.9-44 \%$, mixed studies
\end{abstract}

$3.9-23.8 \%$ and rehabilitation $3.2-11 \%$. The majority of NICU and MICU studies were heterogeneous including different neurovascular diseases, which partly explains the wide range of SAP incidence. The higher incidence in the majority of ICU studies compared to stroke units or acute floor studies is likely explained by the presence of mechanical ventilation, higher stroke severity causing higher rates of aspiration and stroke-induced immunodepression among ICU patients. The short-term mortality of SAP was increased among the mixed and stroke unit studies ranging between 10.1 and $37.3 \%$. SAP was associated with worse functional outcome in the majority of stroke unit and floor studies. Mortality was less consistent among NICU and MICU studies. This difference could be due to the heterogeneity of ICU studies and the effect of small sample size or other independent risk factors for mortality such as the larger neurological deficit, mechanical ventilation, and age, which may simultaneously increase the risk of SAP and mortality confounding the outcomes of SAP itself. The pathophysiology of SAP is likely explained by aspiration combined with stroke-induced immunodepression through complex humeral and neural pathways that include the hypothalamic-pituitary-adrenal axis, parasympathetic and sympathetic systems. Conclusions: A unified definition of SAP, strict inclusion criteria, and the presence of a long-term follow-up need to be applied to the future prospective studies to better identify the incidence and prognosis of SAP, especially among ICU patients.

Copyright $\odot 2013$ S. Karger AG, Basel

Yousef Hannawi, MD

Department of Neurology, Baylor College of Medicine

One Baylor Plaza NB-302

Houston, TX 77030 (USA)

E-Mail yousefhannawi@yahoo.com 


\section{Introduction}

Stroke is a major cause of morbidity and mortality [ 1 , 2]. Medical and neurological complications, including pneumonia, are found to be major causes of death after stroke [3]. Post-stroke pneumonia also increases the financial burden on the medical system. The annual cost of this complication approaches USD 459 million [4] and the average marginal cost of hospitalization is USD 27,633 [5]. These factors reflect the importance of preventing this complication. Major advances have been reached in elucidating the pathophysiological mechanisms of stroke-associated pneumonia (SAP), especially the stroke-induced immunodepression $[6,7]$. In addition, neurological intensive care units (NICUs) have been rapidly expanding in the last few years as, compared to the past, many large ischemic stroke patients are now being managed in specialized NICUs. This may be beneficial in terms of incidence and outcomes of strokerelated complications $[8,9]$. To our knowledge, the incidence and outcomes of SAP after acute ischemic stroke (AIS) have not been thoroughly reviewed among NICU studies. We reviewed studies on the incidence, risk factors, outcomes and prophylaxis of inpatient acute SAP related to AIS according to the study setting with special attention to studies performed in the NICUs. We divided the studies into 5 categories including NICUs, medical intensive care units (MICUs), stroke units, mixed studies combining more than one setting and rehabilitation studies to see if there is any difference according to the study setting. We also reviewed the mechanisms involved in the pathophysiology of SAP including strokeinduced immunodepression which is likely to play a larger role in intensive care unit (ICU) patients where the stroke severity is higher.

\section{Methods}

To determine the incidence, risk factors, microbiology and outcomes of inpatient acute SAP related to AIS, PubMed was searched using the MeSH database for the terms 'stroke' AND 'pneumonia' from January 1, 1978 till September 30, 2012. PubMed was also searched using the terms 'neurology intensive unit' AND 'pneumonia' to include further NICU studies during the same period, as many are heterogeneous and include more than one specific diagnosis. This resulted in 352 papers. Exclusion criteria included non-English literature, animal studies, case reports, papers that only included intracerebral hemorrhage or subarachnoid hemorrhage without AIS data and chronic SAP papers that studied patients after more than 30 days from stroke onset. Thus, studies in long-term facilities and outpatient settings were excluded.
Rehabilitation studies were excluded unless the mean for rehabilitation admission was less than 30 days from stroke onset. This left 54 papers for the final review.

To determine the pathophysiology of stroke-induced immunodepression, we searched the literature in English through PubMed using the term 'stroke-induced immunodepression' for relevant articles in addition to the data extracted from the above search.

\section{Definitions and General Terms}

Post-stroke pneumonia has been used to describe pneumonia that occurs early after stroke [10]. The term 'strokeassociated pneumonia' has been used for the first time by Hilker et al. [11] referring to this concept. SAP is described as early when it happens in the first $72 \mathrm{~h}$ of admission to the hospital. Another classification in use divides SAP into acute (when pneumonia develops within a month of stroke) and chronic (when it occurs later than a month) [10]. Clinical SAP studies used a wide range of criteria to define SAP starting from the Center of Disease Control and Prevention criteria to investigating ventilator-associated pneumonia after stroke to reviewing patients' diagnosis from the charts $[1,5,12]$. We will use the Center of Disease Control and Prevention criteria to define nosocomial pneumonia [13], as they are the most widely used ones among the studies $[14,15]$. According to these criteria, health careassociated pneumonia is classified into 3 categories: clinically defined pneumonia, pneumonia with common bacterial or filamentous fungal pathogens and specific laboratory findings and pneumonia in immunocompromised patients. We will mention the first 2 categories since they are more often related to SAP. Clinically defined pneumonia criteria require the presence of a new and persistent infiltrate or consolidation on at least 1 chest X-ray or at least 2 serial chest $X$-rays in the case of underlying lung disease combined with one of the following clinical signs: fever, leukopenia or leukocytosis and altered mental status in more than 70-year-olds in the absence of other causes. These should be added to 2 of the following signs: newonset purulent sputum or change in the character of the sputum, new-onset or worsening cough, rales, and worsening of gas exchange. Pneumonia with common bacterial pathogen is defined similarly with a positive culture from the blood, pleural fluid, quantitative culture from the bronchoalveolar lavage, or lung parenchyma. However, only one of the last signs (such as new-onset purulent sputum or new-onset cough) is needed instead of two. Ventilatorassociated pneumonia is defined as pneumonia in patients who had a device to control breathing within the 48-hour period before the onset of infection [13]. 


\section{The Pathophysiology of SAP: The Traditional Aspiration Theory and the Newly Described Stroke-Induced Immunodepression}

\section{Aspiration Theory}

Traditionally, SAP is thought to be secondary to aspiration $[10,16,17]$. Aspiration and its related risk factors such as impaired level of consciousness and dysphagia have been found to be important risk factors for SAP across different clinical studies $[11,17,18]$. Many stroke patients have impaired swallowing mechanisms leading to aspiration of oral content during sleep, which may be theoretically related to abnormal dopamine transmission [10]. Experimental evidence for this phenomenon can be found in guinea pigs by blocking $\mathrm{D}_{1}$ dopamine receptors resulting in inhibition of the swallowing reflex and a decrease in substance $P$ in the end organs [19]. A low substance P level in the sputum was also found in elderly patients with aspiration pneumonia [20] and an increase in the serum level of substance $\mathrm{P}$ was observed in a clinical study after treating stroke patients with angiotensin-converting enzyme inhibitor with concomitant resolution of aspiration suggesting a further role of low substance $\mathrm{P}$ in aspiration [21]. However, the higher incidence of pneumonia in stroke patients compared to others who suffer from dysphagia or a compromised level of consciousness [22,23], as well as the predominance of infection in the acute stroke phase when the maximum neurological deficit is present $[24,25]$ suggest that another mechanism is involved in SAP pathogenesis inducing an immunological alteration [26].

\section{Stroke-Induced Immunodepression}

As early as 1974, immunodepression has been thought to accompany AIS [27]. Since then many advances have been achieved elucidating the specific pathophysiological mechanisms. The immunodepression following stroke is the result of activation of 3 systems: the sympathetic system, the parasympathetic system and the hypothalamicpituitary-adrenal axis $[6,7,28]$.

In 2003, Prass et al. [29] provided experimental evidence for neuroendocrine-mediated systemic immunodepression after stroke in a mouse model. Their work showed that stroke induced an apoptotoic loss of lymphocytes, a shift from T-helper (Th) 1 to Th2 cytokine production, spontaneous bacteremia and pneumonia. They also found that blocking the sympathetic activity prevented bacterial infection and the use of propranolol ( $\beta$-blocker) decreased mortality after stroke. The same group later showed in 2006 that nasal inoculation of 200 colony-forming units of Streptococcus pneumoniae was enough to cause pneumonia in a mouse model of cerebral ischemia while 200,000 colonyforming units were required to cause the same disease in sham animals and again pneumonia was preventable by $\beta$-blockade [30]. Currently, there is a large body of experimental and clinical evidence to support peripheral lymphocytopenia and immunological switch from proinflammatory Th1 response to anti-inflammatory Th2 response as a result of sympathetic activation after AIS [31-34]. Sympathetic activation following AIS appears to play a crucial part in stroke-induced immunodepression [6]. These changes are mostly prominent among patients with large strokes $[31,32]$ and they may have a predilection for the stroke location such as the involvement of the insular cortex [35].

There is also evidence for the parasympathetic systemrelated and hypothalamic-pituitary-adrenal-axis-related abnormalities following AIS [36, 37]. Hypothalamic-pituitary-adrenal axis activation results in glucocorticoid secretion from the adrenal gland. Glucocorticoids are known anti-inflammatories and they may cause apoptosis of human T-lymphocytes [38]. High and low circulating cortisol levels are clinically associated with mortality after stroke [36].

Activation of the autonomic parasympathetic centers results in cholinergic activity which suppresses the peripheral cytokine release through macrophage nicotine receptors. This effect has been demonstrated in the adult male Lewis rats by the electric stimulation of the vagal nerve [37]. The neuroendocrine system and the autonomic centers are synchronized through the paraventricular nucleus of the hypothalamus [26]. Thus, these systems function together to induce an immune alteration after stroke $[6,7,28]$.

In summary, SAP is likely a net sum of ongoing aspiration which provides the pathogenic bacteria and immunological alterations as part of the stroke-induced immunodepression [7, 30]. Various comorbidities add to this equation to increase the risk for SAP (fig. 1).

\section{The Pathophysiology of Potential Stroke Worsening as a Result of Infection}

Several mechanisms by which infection or SAP may lead to deterioration of AIS have been proposed. SAP is commonly associated with fever, electrolyte imbalance or hypoxia [26]. These factors may theoretically interfere with the stroke. Fever effects have been extensively investigated in animal models. Fever exacerbates inflammatory cascades resulting in neutrophile accumulation in the injured tissue [39] while therapeutic hypothermia modu- 


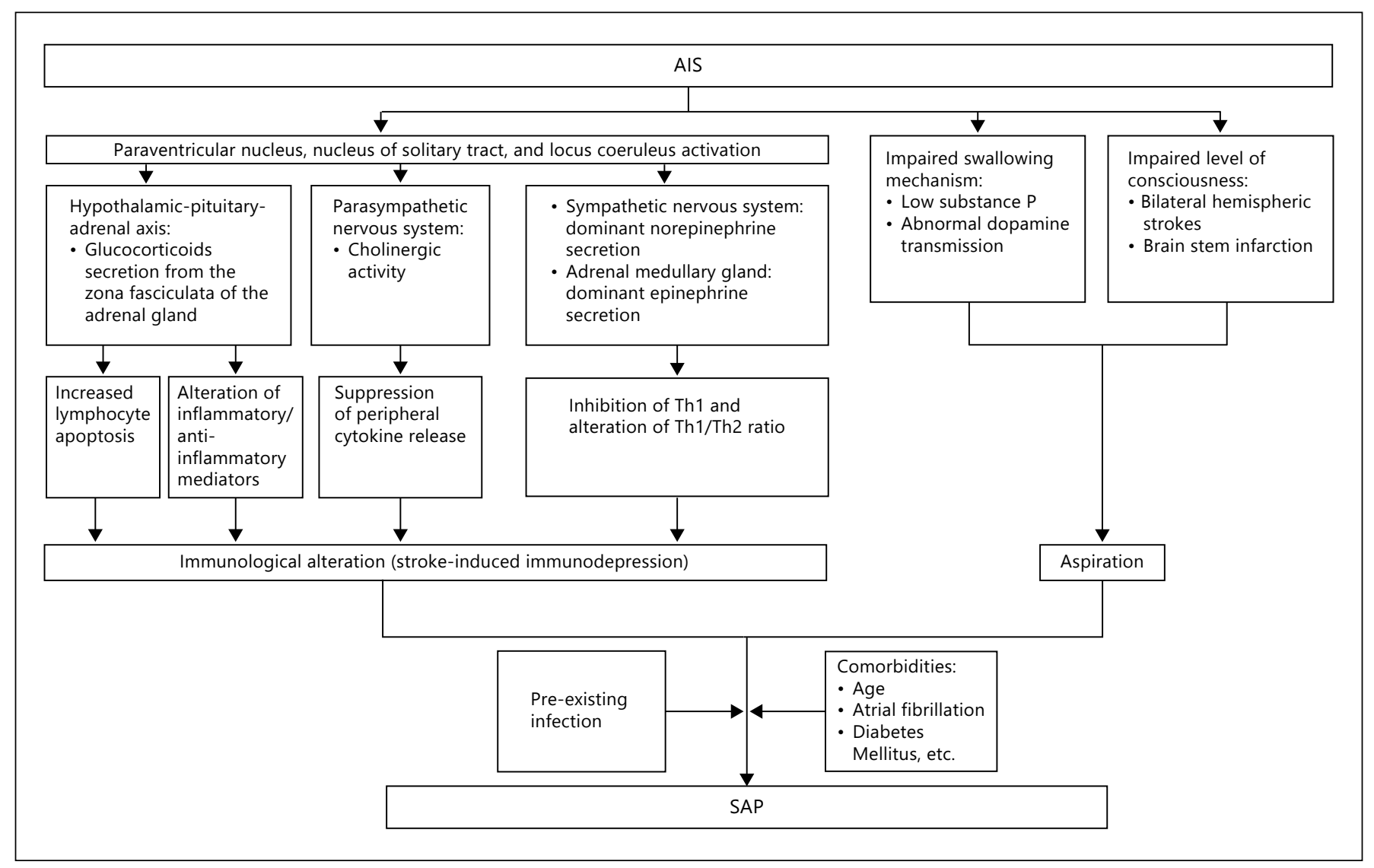

Fig. 1. A scheme demonstrating the pathophysiology of SAP: combining the aspiration theory with the stroke-induced immunodepression theory in addition to the effect of comorbidities.

lates these inflammatory processes [40]. Neuronal excitotoxicity, by increased release of neurotransmitters and free radicals, is another mechanism by which fever may lead to stroke worsening [41]. At the clinical level, a metaanalysis showed that fever is associated with morbidity and mortality after stroke [42]. Electrolyte imbalance, especially hyponatremia, may worsen cerebral edema [43] and hyponatremic stroke patients may have an increased long-term mortality [44]. The entry of bacteria and lipopolysaccharide into the blood stream activates the coagulation as well as fibrinolysis systems and may theoretically result in the extension of the infarcted area [45].

\section{Microbiologic Data}

Since aspiration plays an important role in the pathogenesis of SAP, oral and nasopharyngeal pathogens are expected to be encountered frequently [10]. The oral flora of stroke patients is rapidly altered after stroke and aerobic Gram-negative bacilli colonize in the stroke patients' mouths more often than in normal people [46]. Enterobacter sakazakii is another example of organisms that can be isolated from the mouth of stroke patients and are rarely found otherwise [47]. Thus, aerobic Gram-negative bacilli are expected to be the most encountered pathogens. Overall, cultures often remain negative without bacterial growth $[15,48]$. Staphylococcus aureus which resides in the nasal cavity, and aerobic Gram-negative bacilli including Klebsiella pneumoniae, Pseudomonas aeruginosa and Acinetobacter baumannii were the most frequently isolated pathogens in the studies identified by our search $[11,14,15,23,48-51]$. These findings are consistent with the results of a recent meta-analysis of strokeassociated infections which often showed negative cultures or the same above-isolated pathogens in addition to Escherichia coli and Enterococcus species, while Streptococcus species were occasionally isolated [52]. Thus, the 
Table 1. NICU studies of SAP

\begin{tabular}{|c|c|c|c|c|}
\hline Study & Incidence & Major risk factors & Prognosis & Study baseline characteristics \\
\hline $\begin{array}{l}\text { Sui and } \\
\text { Zhang } \\
{[53]}\end{array}$ & $37.98 \%$ & $\begin{array}{l}\text { Older age, tracheal intubation, } \\
\text { decreased level of consciousness } \\
\text { and nasal feeding }\end{array}$ & - & $\begin{array}{l}\text { Retrospective; } \mathrm{n}=1,435 \\
\text { pAIS }=64.4 \% ; \mathrm{mA}=65.7 \text { years; } \\
\text { mNIHSS }=7.5 ; \mathrm{pMV}=2.2 \%\end{array}$ \\
\hline $\begin{array}{l}\text { Yeh } \\
\text { et al. [54] }\end{array}$ & $56.6 \%$ & $\begin{array}{l}\text { Older age and higher NIHSS } \\
\text { score, while dysphagia screening } \\
\text { decreased SAP risk }\end{array}$ & $\begin{array}{l}\text { SAP was not associated } \\
\text { with in-hospital mortality } \\
\text { on multivariate analysis }\end{array}$ & $\begin{array}{l}\text { Consecutive series } \mathrm{n}=176 ; \\
\text { pAIS }=68.8 \% ; \mathrm{mA}=67.5 \text { years; } \\
\text { mNIHSS }=14.3 ; \mathrm{pMV}=11.3 \%\end{array}$ \\
\hline $\begin{array}{l}\text { Josephson } \\
\text { et al. [14] }\end{array}$ & $4.1 \%$ & $\begin{array}{l}\text { Younger age, longer hours of } \\
\text { ventilation, and transfer outside } \\
\text { the unit for imaging studies }\end{array}$ & $\begin{array}{l}\text { Increased hours of ventilation } \\
\text { but no difference in mortality }\end{array}$ & $\begin{array}{l}\text { Retrospective; } \mathrm{n}=585 \\
\mathrm{~mA}=58.6 \text { years; } \mathrm{pMV}=100 \%\end{array}$ \\
\hline $\begin{array}{l}\text { Yan } \\
\text { et al. [56] }\end{array}$ & $45.9 \%$ & - & - & $\begin{array}{l}\text { Retrospective } ; \mathrm{n}=137 \\
\text { pAIS }=54 \% ; \mathrm{mA}=70.96 \text { years; } \\
\text { pMV }=27 \%\end{array}$ \\
\hline $\begin{array}{l}\text { Yilmaz } \\
\text { et al. [50] }\end{array}$ & $27 \%$ & $\begin{array}{l}\text { Age }>70 \text { years, central venous } \\
\text { catheterization and parenteral } \\
\text { nutrition }\end{array}$ & - & $\begin{array}{l}\text { Prospective; } \mathrm{n}=171 ; \mathrm{pAIS}= \\
68.1 \% ; \mathrm{mA}=66 \text { years; } \mathrm{pMV}=50 \% \\
\text { among the infected group }\end{array}$ \\
\hline $\begin{array}{l}\text { Commichau } \\
\text { et al. [55] }\end{array}$ & $9.5 \%$ & - & - & $\begin{array}{l}\text { Prospective; } \mathrm{n}=387 ; \mathrm{pAIS}=6 \% \\
\mathrm{~mA}=54 \text { years; } \mathrm{pMV}=19.6 \%\end{array}$ \\
\hline $\begin{array}{l}\text { Hilker } \\
\text { et al. [11] }\end{array}$ & $21.4 \%$ & $\begin{array}{l}\text { Dysphagia, MV, abnormal chest } \\
\text { X-ray, vertebrobasilar and } \\
\text { multihemispheric strokes }\end{array}$ & $\begin{array}{l}\text { In-hospital mortality was } \\
26.9 \% .14 \text {-month follow-up } \\
\text { mortality was } 35.3 \% .14 \text {-month } \\
\text { functional outcome was worse, } \\
\text { mean mRS score }=3.5\end{array}$ & $\begin{array}{l}\text { Prospective; } \mathrm{n}=124 \\
\text { pAIS }=100 \% ; \mathrm{mA}=63.8 \text { years; } \\
\text { pMV }=13 \%\end{array}$ \\
\hline
\end{tabular}

Includes NICU studies of SAP listed in chronological order starting from the most recent one and summarizing the incidence, risk factors, prognosis, and study baseline characteristics including: study design, sample size (n), proportion of acute ischemic stroke of the sample (pAIS), mean Age (mA), stroke severity represented by the mean baseline NIHSS of the study sample (mNIHSS) and mechanical ventilation proportion of the sample $(\mathrm{pMV})$ when these variables are reported. $-=$ Not studied or mentioned; $\mathrm{mRS}=$ modified Rankin Scale.

pattern of SAP bacteriology is most consistent with earlyonset nosocomial pneumonia or community-acquired aspiration syndrome likely related to aspiration at the time of stroke ictus [52].

\section{Incidence of SAP}

Studies identified by our search methodology are summarized in tables 1-5. These studies are divided into 5 categories according to the setting in which they were performed: NICUs (table 1), MICUs (table 2), stroke units (table 3), mixed studies (table 4), and rehabilitation units (table 5). Mixed studies include the ones which were performed in more than one acute setting such as the different acute floors, or when the acute settings were not specified.

The incidence of SAP among the majority of NICU studies ranged between 9.5 and $56.6 \%[11,15,50,51,53$, 54] except for one study in which the incidence was $4.1 \%$ [14]. However, this study included all types of neurovascular patients in addition to stroke patients and 
Table 2. MICU studies of SAP

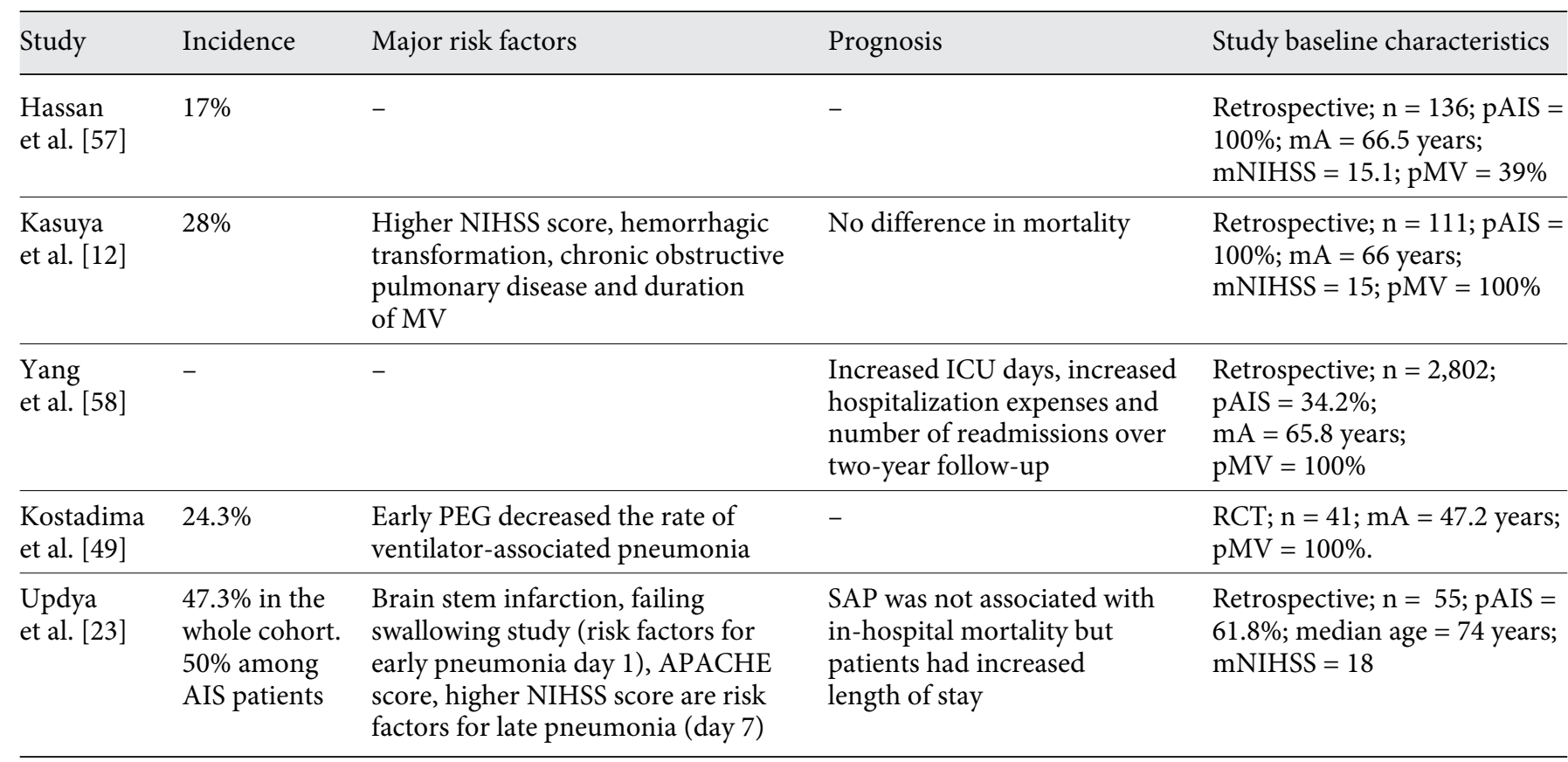

Includes MICU studies of SAP listed in chronological order starting from the most recent one and summarizing the incidence, risk factors, prognosis, and study baseline characteristics including: study design, sample size (n), proportion of acute ischemic stroke of the sample (pAIS), mean age (mA), stroke severity represented by the mean baseline NIHSS of the study sample (mNIHSS) and mechanical ventilation proportion of the sample (pMV) when these variables are reported. - = Not studied or mentioned; APACHE $\mathrm{II}=$ acute physiology and chronic health evaluation; RCT = randomized clinical trial; PEG = percutaneous gastrostomy.

they were younger (mean age 58.6 years). The occurrence was much higher among febrile patients (40.2$70.8 \%)[55,56]$ reflecting the importance of SAP as a cause of fever after stroke. Among MICU studies, this incidence has ranged between 17 and 50\% [12, 23, 49, $57,58]$ and appeared to be similar to NICU studies. The majority of SAP studies have been performed in stroke units or in mixed acute settings. The incidence of SAP among the majority of studies that have been performed exclusively in stroke units ranged between 3.9 and $12 \%$ $[1,3,17,24,59-67]$ except for one study where the incidence was $44 \%$ [25]. This could reflect a selection bias since all patients included in this study had nasogastric tube feeding, higher rate of mechanical ventilation (18\%) and their strokes were more severe [mean baseline National Institutes of Health Stroke Scale (NIHSS) score $=13.4]$. Studies performed in mixed acute settings found an incidence between 3.9 and $23.8 \%$ [4, 5, 9, 16, $18,48,68-84]$, while the incidence among selected rehabilitation studies ranged between 3.2 and $11 \%$ [69, 85-87].

Stroke-Associated Pneumonia
It is hard to make a comparison among these studies because they are highly heterogeneous, especially those performed in critical care settings. The majority of ICU studies included intracerebral hemorrhage or subarachnoid hemorrhage in addition to AIS [14, 51, 54]. Some have included AIS alone, the majority of which were performed in stroke units or acute general floors $[1,11,67$, 70]. There are also differences in the methods of defining SAP (chart review [64], discharge diagnosis codes [5], or through Center of Disease Control and Prevention criteria [11]), the rates of mechanically ventilated patients vary as well $[12,25,66]$, in addition to the broad geographic distribution of these studies $[24,48,50]$. Overall, ICU studies are smaller and more heterogeneous compared to floor or stroke unit studies (pooled sample size NICU: 3,756; MICU: 3,145; stroke units: 45,829; mixed studies: 257,232 , and rehabilitation: 1,400 ). The incidence of SAP appears to be similar in NICUs and MICUs and higher than that in the stroke units or acute general floors. These findings are consistent with the results of a recent meta-analysis [52]. This is likely 
Table 3. Stroke unit studies of SAP

\begin{tabular}{|c|c|c|c|c|}
\hline Study & Incidence & Major risk factors & Prognosis & Study baseline characteristics \\
\hline $\begin{array}{l}\text { Ifejika-Jones } \\
\text { et al. [64] }\end{array}$ & $5.4 \%$ & $\begin{array}{l}\text { Male sex and higher } \\
\text { NIHSS score }\end{array}$ & $\begin{array}{l}\text { Increased mortality }(13.23 \%) \text {, } \\
\text { and less likely to be discharged } \\
\text { home }\end{array}$ & $\begin{array}{l}\text { Retrospective; } \mathrm{n}=4,971 \\
\text { pAIS }=55.26 \% ; \mathrm{mA}=63 \text { years; } \\
\text { mNIHSS }=6\end{array}$ \\
\hline $\begin{array}{l}\text { Ingeman } \\
\text { et al. [65] }\end{array}$ & $9 \%$ & - & $\begin{array}{l}30 \text {-day mortality was } 24.9 \% \\
\text { (adjusted risk 1.59), and 1-year } \\
\text { mortality } 50.9 \% \text { (adjusted risk } \\
1.76 \text { ) }\end{array}$ & $\begin{array}{l}\text { Prospective } \mathrm{n}=13,721 \\
\text { pAIS }=79.4 \% ; \mathrm{mA}=73 \text { years }\end{array}$ \\
\hline $\begin{array}{l}\text { Koennecke } \\
\text { et al. [3] }\end{array}$ & $7.7 \%$ & - & $\begin{array}{l}7 \text {-day in-hospital death was } \\
\text { increased }(12.2 \%) \text { and poor } \\
\text { functional outcome at discharge } \\
(\mathrm{mRS}>3, \mathrm{OR}=24.7)\end{array}$ & $\begin{array}{l}\text { Consecutive series; } \mathrm{n}=16,518 \\
\mathrm{pAIS}=92.7 \% ; \mathrm{mA}=71.15 \text { years }\end{array}$ \\
\hline $\begin{array}{l}\text { Minnerup } \\
\text { et al. [66] }\end{array}$ & $12 \%$ & Stroke size & - & $\begin{array}{l}\text { Prospective; } \mathrm{n}=591 ; \mathrm{mA}=67.7 \text { years; } \\
\text { mNIHSS }=6.9 ; \mathrm{pMV}=70 \%\end{array}$ \\
\hline $\begin{array}{l}\text { Saposnik } \\
\text { et al. [67] }\end{array}$ & $6.6 \%$ & - & - & $\begin{array}{l}\text { Prospective } \mathrm{n}=3,631 ; \mathrm{pAIS}=100 \% \\
\text { mean Canadian Neurological Scale } \\
\text { score }=8\end{array}$ \\
\hline $\begin{array}{l}\text { Vermeij } \\
\text { et al. [1] }\end{array}$ & $7.5 \%$ & - & $\begin{array}{l}\text { Mortality was }(49 \%, \mathrm{HR}=2.1) \text { at } \\
1 \text { year. Poor outcome at } 1 \text { year } \\
(\mathrm{OR}=10)\end{array}$ & $\begin{array}{l}\text { Consecutive series } ; \mathrm{n}=521 \\
\text { pAIS }=100 \% ; \mathrm{mA}=70.5 \text { years; } \\
\text { median } \mathrm{BI}=10\end{array}$ \\
\hline $\begin{array}{l}\text { Dziedzek } \\
\text { et al. [61] }\end{array}$ & $11.7 \%$ & - & - & $\begin{array}{l}\text { Retrospective; } \mathrm{n}=689 ; \mathrm{mA}=69.6 \text { years; } \\
\text { pAIS }=100 \% ; \text { mean Scandinavian } \\
\text { Stroke Scale score }=30.9\end{array}$ \\
\hline $\begin{array}{l}\text { Dziewas } \\
\text { et al. [25] }\end{array}$ & $44 \%$ & $\begin{array}{l}\text { Duration of nasogastric } \\
\text { tube feeding, endotracheal } \\
\text { intubation, aphasia and } \\
\text { higher NIHSS score }\end{array}$ & $\begin{array}{l}\text { SAP was not associated with } \\
\text { poor functional outcome at } \\
3 \text { months on multivariate } \\
\text { analysis }\end{array}$ & $\begin{array}{l}\text { Prospective } \mathrm{n}=100 \\
\mathrm{~mA}=68.8 \text { years } ; \mathrm{mNIHSS}=13.4 \\
\mathrm{pMV}=18 \%\end{array}$ \\
\hline $\begin{array}{l}\text { Broadley } \\
\text { et al. [59] }\end{array}$ & $5 \%$ & Dysphagia & - & $\begin{array}{l}\text { Prospective; } \mathrm{n}=149 ; \mathrm{pAIS}=81 \% \\
\mathrm{~mA}=68.3 \text { years } ; \mathrm{mBI}=45.7\end{array}$ \\
\hline $\begin{array}{l}\text { Weimer } \\
\text { et al. [24] }\end{array}$ & $\begin{array}{l}7.4 \% \text { in the } \\
1 \text { st week, } \\
\text { then } 1.8 \%\end{array}$ & $\begin{array}{l}\text { Older age, higher NIHSS } \\
\text { score, prior stroke, and } \\
\text { atrial fibrillation }\end{array}$ & - & $\begin{array}{l}\text { Prospective; } \mathrm{n}=3,866 ; \mathrm{pAIS}=100 \% \\
\mathrm{~mA}=66.6 \text { years; } \mathrm{mNIHSS}=7.8\end{array}$ \\
\hline $\begin{array}{l}\text { Lim } \\
\text { et al. [17] }\end{array}$ & $10 \%$ & Aspiration & - & Prospective; $\mathrm{n}=50 ; \mathrm{mA}=67.5$ years \\
\hline
\end{tabular}

Includes stroke units' studies of SAP listed in chronological order starting from the most recent one and summarizing the incidence, risk factors, prognosis, and study baseline characteristics including: study design, sample size (n), proportion of acute ischemic stroke of the sample (pAIS), mean age (mA), stroke severity represented by the mean baseline NIHSS of the study sample (mNIHSS) and mechanical ventilation proportion of the sample (pMV) when these variables are reported. - = Not studied or mentioned; HR = hazard ratio; $\mathrm{mRS}=$ modified Rankin Scale; $\mathrm{OR}=$ odds ratio; $\mathrm{RCT}=$ randomized clinical trial. 
Table 4. SAP studies performed in different mixed settings

\begin{tabular}{|c|c|c|c|c|}
\hline Study & Incidence & Risk factors & Prognosis & Study baseline characteristics \\
\hline Chen et al. [69] & $\begin{array}{l}\text { Acute ward } 23.8 \% \text {, } \\
\text { rehabilitation } 6.7 \%\end{array}$ & - & May prolong the length of stay & $\begin{array}{l}\text { Retrospective; } \mathrm{n}=341 \\
\text { pAIS }=66.2 \% ; \mathrm{mA}=73.4 \text { years }\end{array}$ \\
\hline Wilson [5] & $8.1 \%$ & $\begin{array}{l}\text { Older age, CHF, COPD, } \\
\text { and DM }\end{array}$ & Mortality was increased (20\%) & $\begin{array}{l}\text { Retrospective; } \mathrm{n}=183,976 \\
\mathrm{~mA}=73.3 \text { years }\end{array}$ \\
\hline Finlayson et al. [73] & $7.1 \%$ & $\begin{array}{l}\text { Older age, male sex, stroke } \\
\text { severity and COPD }\end{array}$ & $\begin{array}{l}\text { Increased } 30 \text {-day and } 1 \text {-year } \\
\text { mortality ( } 37.3 \% \text { and } 60.1 \% \text {, re- } \\
\text { spectively) and worse functional } \\
\text { outcome (mRS }>3 \text { in } 12.3 \%)\end{array}$ & $\begin{array}{l}\text { Retrospective; } \mathrm{n}=8,251 ; \\
\mathrm{mA}=72.2 \text { years; } \mathrm{pAIS}=100 \% \\
\text { Canadian Neurological Scale } \\
\text { score }=7.7\end{array}$ \\
\hline Jones et al. [79] & $7.3 \%$ & $\begin{array}{l}\text { Shift changes in the } \\
\text { emergency room }\end{array}$ & - & $\begin{array}{l}\text { Retrospective; } \mathrm{n}=336 ; \\
\text { pAIS }=100 \% ; \mathrm{mA}=65 \text { years; } \\
\text { mNIHSS }=5.8\end{array}$ \\
\hline Ota et al. [81] & $21.9 \%$ & Secretion severity & - & $\begin{array}{l}\text { Retrospective; } \mathrm{n}=72 ; \\
\text { pAIS }=73.6 \% ; \mathrm{mA}=74.2 \text { years }\end{array}$ \\
\hline Chumbler et al. [70] & $10.4 \%$ & $\begin{array}{l}\text { Older age, higher NIHSS, } \\
\text { dysphagia and past history } \\
\text { of pneumonia }\end{array}$ & - & $\begin{array}{l}\text { Retrospective; } \mathrm{n}=1,363 ; \\
\text { pAIS }=100 \% ; \text { median } \\
\text { age }=71.4 \text { years; NIHSS }=9.4\end{array}$ \\
\hline Cuesy et al. [71] & $19.7 \%$ & $\begin{array}{l}\text { Passive turning and } \\
\text { mobilization program } \\
\text { decreases the risk }\end{array}$ & - & $\begin{array}{l}\text { RCT } ; n=223 ; \mathrm{mA}=73.2 \text { years; } \\
\text { pAIS }=100 \% ; \mathrm{pMV}=7.6 \%\end{array}$ \\
\hline $\begin{array}{l}\text { Lakshminarayan } \\
\text { et al. [18] }\end{array}$ & $3.9 \%$ & $\begin{array}{l}\text { Older age, altered level of } \\
\text { consciousness, and failing } \\
\text { dysphagia screening }\end{array}$ & $\begin{array}{l}\text { Mortality was increased }(10.1 \%) \\
\text { and length of stay }\end{array}$ & $\begin{array}{l}\text { Retrospective; } \mathrm{n}=18,017 ; \\
\text { pAIS }=83.6 \% ; \text { median } \\
\text { age }=71 \text { years; } \text { pMV }=0 \%\end{array}$ \\
\hline Kolb and Broker [84] & $14 \%$ & Aspiration & - & $\begin{array}{l}\text { Consecutive series; } \mathrm{n}=50 ; \\
\text { median age }=78 \text { years }\end{array}$ \\
\hline Hong et al. [77] & $12 \%$ & $\begin{array}{l}\text { Older age, higher NIHSS, } \\
\text { mRS } 3-5 \text {, and low baseline } \\
\text { hemoglobin }\end{array}$ & $\begin{array}{l}29.5 \% \text { had poor outcome at } \\
3 \text { months (adjusted OR } 4.44 \text { ) }\end{array}$ & $\begin{array}{l}\text { Prospective; } \mathrm{n}=1,254 ; \\
\mathrm{pAIS}=100 \% ; \mathrm{mA}=66.5 \text { years; } \\
\text { median NIHSS }=4\end{array}$ \\
\hline Katzan et al. [4] & $5.6 \%$ & $\begin{array}{l}\text { Older age, increased stroke } \\
\text { severity and admission } \\
\text { from nursing home }\end{array}$ & $\begin{array}{l}30 \text {-day mortality was } 26.5 \% \text { and } \\
\text { increased length of hospital stay }\end{array}$ & $\begin{array}{l}\text { Retrospective; } \mathrm{n}=11,286 ; \\
\text { pAIS }=91.75 \% ; \mathrm{mA}=76.8 \text { years }\end{array}$ \\
\hline Sellars et al. [82] & $19 \%$ & $\begin{array}{l}\text { Older age, dysarthria, } \\
\mathrm{mRS}>2 \text {, and failing } \\
\text { water swallow }\end{array}$ & - & $\begin{array}{l}\text { Prospective; } \mathrm{n}=412 ; \\
\text { pAIS }=94.9 \% ; \mathrm{mA}=67.9 \text { years; } \\
\text { median NIHSS }=5\end{array}$ \\
\hline Hassan et al. [48] & $23 \%$ & - & - & $\begin{array}{l}\text { Retrospective; } \mathrm{n}=443 ; \\
\text { pAIS }=57 \% ; \mathrm{mA}=58 \text { years }\end{array}$ \\
\hline Huang et al. [16] & $19.7 \%$ & $\begin{array}{l}\text { Feeding by trained nurse } \\
\text { decreased SAP compared } \\
\text { to family feeding }\end{array}$ & - & Prospective; $\mathrm{n}=96$ \\
\hline Kwon et al. [9] & $16.4 \%$ & $\begin{array}{l}\text { Higher NIHSS score, older } \\
\text { age, dysphagia and MV }\end{array}$ & - & $\begin{array}{l}\text { Retrospective; } \mathrm{n}=286 ; \\
\text { pAIS }=80.4 \% ; \mathrm{mA}=62.8 \text { years; } \\
\text { mNIHSS }=12.6\end{array}$ \\
\hline $\begin{array}{l}\text { Hanchaiphilboolkul } \\
\text { [83] }\end{array}$ & $4.3 \%$ & $\begin{array}{l}\text { Atrial fibrillation, large- } \\
\text { vessel disease, altered level } \\
\text { of consciousness and } \\
\text { previous stroke }\end{array}$ & - & $\begin{array}{l}\text { Retrospective; } \mathrm{n}=332 \\
\text { pAIS }=100 \%\end{array}$ \\
\hline Hinchey et al. [76] & $4.5 \%$ & $\begin{array}{l}\text { Older age, higher NIHSS } \\
\text { score, while formal } \\
\text { dysphagia screening } \\
\text { decreases the risk }\end{array}$ & $\begin{array}{l}\text { Mortality was increased } \\
\text { ( } 5.4 \text { times, } 21 \% \text { ), in addition to } \\
\text { increased length of stay }\end{array}$ & $\begin{array}{l}\text { Prospective; } \mathrm{n}=2,532 ; \\
\text { pAIS }=100 \% ; \text { age }=70.5 \text { years; } \\
\text { mNIHSS }=7.2\end{array}$ \\
\hline Aslanyan et al. [68] & $\begin{array}{l}10.9 \% \text { in the } \\
1 \text { st week } \\
\text { then } 0.5 \%\end{array}$ & $\begin{array}{l}\text { Older age, higher NIHSS } \\
\text { score, male sex and history } \\
\text { of DM }\end{array}$ & $\begin{array}{l}\times 2.2 \text { increase in short-term } \\
\text { mortality. Poor outcome at } \\
3 \text { months (OR 3.4) }\end{array}$ & $\begin{array}{l}\text { Prospective; } \mathrm{n}=1,455 ; \\
\text { pAIS }=100 \% ; \mathrm{mA}=70 \text { years; } \\
\text { mNIHSS }=13\end{array}$ \\
\hline
\end{tabular}


Table 4. (continued)

\begin{tabular}{|c|c|c|c|c|}
\hline Study & Incidence & Risk factors & Prognosis & Study baseline characteristics \\
\hline Field et al. [72] & $4.7 \%$ & - & - & $\begin{array}{l}\text { Retrospective; } n=11,642 \\
\text { pAIS = } 69.5 \%\end{array}$ \\
\hline Hamidon et al. [75] & $12.3 \%$ & $\begin{array}{l}\text { Glasgow Coma Scale less } \\
\text { than } 9, \mathrm{BI}<5 \text {, and middle } \\
\text { cerebral artery stroke }\end{array}$ & $\begin{array}{l}\text { Mortality was increased } \\
\text { (OR 14.83) }\end{array}$ & $\begin{array}{l}\text { Consecutive series; } \mathrm{n}=163 ; \\
\mathrm{pAIS}=100 \% ; \mathrm{mA}=62.2 \text { years; } \\
\text { mean } \mathrm{BI}=10.1\end{array}$ \\
\hline Grau et al. [74] & $9.2 \%$ & - & - & $\begin{array}{l}\text { Consecutive series; } \mathrm{n}=130 \\
\text { pAIS }=100 \% ; \mathrm{mA}=61 \text { years; } \\
\text { mNIHSS = } 7.75\end{array}$ \\
\hline Johnston et al. [78] & $5 \%$ & - & $\begin{array}{l}\text { Serious medical event was } \\
\text { associated with poor functional } \\
\text { outcome (OR } 4.4 \text { on Glasgow } \\
\text { Outcome Scale) }\end{array}$ & $\begin{array}{l}\text { Prospective; } \mathrm{n}=279 \\
\text { pAIS }=100 \% ; \mathrm{mA}=69 \text { years; } \\
\text { median NIHSS = } 9\end{array}$ \\
\hline
\end{tabular}

Includes mixed studies of SAP performed in multiple acute settings listed in chronological order starting from the most recent one and summarizing the incidence, risk factors, prognosis, and study baseline characteristics including: study design, sample size (n), proportion of acute ischemic stroke of the sample (pAIS), mean age (mA), stroke severity represented by the mean baseline NIHSS of the study sample (mNIHSS) and mechanical ventilation proportion of the sample (pMV) when these variables are reported. $-=$ Not studied or mentioned; $\mathrm{BI}=\mathrm{Barthel}$ index; $\mathrm{CHF}=$ congestive heart failure; $\mathrm{COPD}=$ chronic obstructive lung disease; $\mathrm{DM}=$ diabetes mellitus; $\mathrm{mRS}=$ modified Rankin Scale; $\mathrm{MV}=$ mechanical ventilation; $\mathrm{OR}=$ odds ratio; $\mathrm{RCT}=$ randomized clinical trial.

Table 5. SAP studies performed on rehabilitation wards

\begin{tabular}{lllll}
\hline Study & Incidence & Risk factors & Prognosis & Study baseline characteristics \\
\hline Falsetti et al. [85] & $5.9 \%$ & Dysphagia & - & Consecutive series; $\mathrm{n}=151 ; \mathrm{pAIS}=74.1 \% ; \mathrm{mA}=79.4$ years \\
Lipson et al. [86] & $3.2 \%$ & - & - & Retrospective; $\mathrm{n}=819 ; \mathrm{pAIS}=86.5 \% ; \mathrm{mA}=65.9$ years \\
Teasell et al. [87] & $11 \%$ & - & - & Retrospective; $\mathrm{n}=89 ; \mathrm{pAIS}=82 \% ; \mathrm{mA}=61.9$ years
\end{tabular}

Includes studies of SAP performed on acute rehabilitation wards listed in chronological order starting from the most recent one and summarizing the incidence, risk factors, prognosis, and study baseline characteristics including: study design, sample size (n), proportion of acute ischemic stroke of the sample (pAIS), mean age (mA) when these variables are reported. - = Not studied or mentioned.

explained by the effect of comorbidities, mechanical ventilation, and higher stroke severity among critical care unit patients causing higher rates of aspiration and immunological alteration as part of the stroke-induced immunodepression.

\section{Prognosis of SAP}

The outcome of each specific study is listed in tables $1-5$. It is hard to make comparisons for the same reasons mentioned in the incidence section in addition to the large differences in the follow-up period among these studies $[1,5,80]$. Using different functional scales such as the Barthel Index or the modified Rankin Scale for assessment of the patient's functional outcome may add to this difficulty $[25,68]$. Overall, the majority of studies performed in the stroke units and acute hospital floors report SAP as an independent risk factor for mortality after AIS $[1,64,65,68,73,80]$. The shortterm mortality at 30 days or time of discharge ranged between 10.1 and $37.3 \%$ [ $3,5,18,64,65,73,77,80]$, while long-term mortality was $49-60.1 \%[1,65,73]$. SAP was also associated with poor functional outcome 
Table 6. Prophylactic measures against SAP

Possible mechanism of action

\begin{tabular}{ll}
\hline $\begin{array}{l}\text { Established methods } \\
\text { Nil per os status on } \\
\text { admission }\end{array}$ & $\begin{array}{l}\text { Decreases aspiration by targeting } \\
\text { dysphagia }\end{array}$ \\
\hline $\begin{array}{l}\text { Institution of formal } \\
\text { dysphagia screening } \\
\text { protocol }\end{array}$ & $\begin{array}{l}\text { Identifies patients at risks for } \\
\text { aspiration by swallowing } \\
\text { assessment }\end{array}$ \\
\hline $\begin{array}{l}\text { Avoidance of invasive } \\
\text { procedures }\end{array}$ & $\begin{array}{l}\text { Decreases the risk of bacteremia } \\
\text { and bacterial colonization }\end{array}$ \\
\hline
\end{tabular}

Early mobilization $\quad$ May decrease the risk of aspiration

Areas for future research

Cilostazol May improve swallowing reflex by working on dopamine and substance $\mathrm{P}$ through increasing local circulation

Prophylactic antibiotics administration

May decrease bacterial propagation to infection

\begin{tabular}{ll}
\hline $\begin{array}{l}\text { Angiotensin-converting } \\
\text { enzyme inhibitors }\end{array}$ & $\begin{array}{l}\text { May improve swallowing mecha- } \\
\text { nism by increasing substance P }\end{array}$ \\
\hline Propranolol & $\begin{array}{l}\text { Targets early lymphocyte activa- } \\
\text { tion by blocking the sympathetic } \\
\text { activity }\end{array}$ \\
\hline
\end{tabular}

Caspase inhibitor

Q-VD-OPH

Targets lymphocyte apoptosis induced by the stroke

Summarizes the current established and controversial methods for prophylaxis against SAP with their mechanisms of action. in multiple studies $[1,3,63,68,73,77,78]$. However, this was not the case in one study after correcting for other independent factors which can be explained by the small sample size $(n=100)$, preselection of patients with dysphagia and nasogastric tube feeding and high rates of mechanically ventilated patients (18\%) [25]. On the other hand, the data regarding outcome of SAP in the critical care units are inconsistent. Hilker et al. [11] showed higher in-hospital mortality (26.9\%), higher mortality at 14 months of follow-up (35.3\%) and worse functional outcome at 14 months. The rest of the identified ICU studies did not associate SAP with mortality $[12,14,23,54,57]$. This could be due to the heterogeneity of these studies and the effects of selection bias, small sample size [23] and lack of long-term follow-up [12]. However, the effect of other independent risk factors for outcome, such as high NIHSS score [25], fever
[88] and mechanical ventilation, needs to be deeply investigated among ICU studies. These factors may simultaneously increase the patient's risk for SAP, mortality and poor functional outcome confounding the outcomes of SAP itself.

\section{Risk Factors for SAP}

Risk factors identified in each study are listed in tables 1-5. Overall, stroke severity measured by the NIHSS or the modified Rankin Scale is a major independent risk factor for SAP [12, 15, 24, 54, 64, 66, 70, 77]. Dysphagia and aspiration are other risk factors, which have frequently been reported in multiple studies $[9,11,15,17$, 59], as well as old age $[4,18,24,53,54,70,73]$. Other risk factors reported by various studies include: mechanical ventilation $[9,11,15]$, APACHE II score/organ failure status [12], male sex $[9,64,68]$, left anterior cerebral artery stroke or size of the lesion more than $1 / 3$ of the middle cerebral artery territory [66], brain stem infarction $[11,23]$, multihemispheric infarction [11], nonlacunar basal ganglia infarct [15], atrial fibrillation [24, 83], admission from a nursing home [80], dysarthria [9, 82], altered level of consciousness, coma or abnormal pupillary exam $[15,18,80]$. Diabetes or hyperglycemia on admission, congestive heart failure, chronic obstructive pulmonary disease or smoking, history of pneumonia and low albumin blood level $[5,12,68,70,77]$, and the presence of other types of infection at the time of admission such as urinary tract infection [15] have all been found to increase the risk for SAP.

\section{Prophylaxis of SAP}

SAP is a complex disease and multiple factors are involved in the pathogenesis as it has been mentioned. Successful prophylactic measures should target these different pathophysiological steps (table 6).

The most widely accepted measure during the management of acute stroke is the nil per os status on admission to avoid overt aspiration till swallowing is checked [89]. The institution of a formal dysphagia screening protocol, including a water swallow test, significantly decreases the risk of pneumonia $[63,76]$. Enteric feeding methods such as a percutaneous gastrostomy tube or a nasal feeding tube are applied to patients who continue to exhibit signs of unsafe swallowing. These methods do not eliminate the occurrence of pneumonia since aspiration 
of oral content may continue [25]. A recent Cochrane review of the clinical trials showed no difference between the two methods regarding the occurrence of pneumonia in patients with dysphagia; however, percutaneous gastrostomy tube was safer and more effective in terms of feeding [90]. Early mobilization is recommended as it decreases the risk for SAP [71].

Administration of antibiotics in the acute period following stroke remains a hotly debated topic. Moxifloxacin decreased the incidence of SAP and mortality and improved outcome in an experimental mouse model for stroke [91]. Using this antibiotic in a clinical trial in humans (PANTHERIS) decreased infection rate as per the protocol analysis. However, it failed to show a benefit in the intention-to-treat analysis and it did not affect outcomes or survival [92], and another clinical trial using levofloxacin failed to show a decrease in the infection rate [93]. More recently, the Mannheim Infection in Stroke Study showed that patients in the intervention group, who were administered mezlocillin plus sulbactam over 4 days, had a decrease in body temperature and lower rates of infection, which may be associated with better clinical outcome [94]. The most recently published meta-analysis of clinical trials using prophylactic antibiotics after stroke did show a decrease in the infection rate without any effect on mortality or dependency [95]. To further investigate this matter, the Preventive Antibiotics in Stroke Study, a large randomized clinical trial involving 3,200 patients, has been started. This trial will use $2 \mathrm{~g}$ of ceftriaxone as a preventative antibiotic for 4 days. The primary outcomes will be the functional outcomes at 3 months. The rest of the secondary outcomes will involve death at discharge and at 3 months, infection rates during hospitalization and length of stay [96].

Another controversial method is decontamination of the digestive tract in stroke patients aiming to decrease the bacteria colonizing in the mouth and eventually decrease SAP. This method had an effect on SAP incidence in a small study without changing the mortality or morbidity of these patients [62].

Targeting the pathophysiological steps involved in the stroke-induced immunodepression provides an interesting area for research. Propranolol, a $\beta$-blocker, has been used experimentally in a mouse model to block the sympathetic activity which induces an apoptotic lymphocytic loss and alteration in the Th1/Th2 ratio. This decreased post-stroke pneumonia and mortality in mice [29]. Another experimental method targets stroke-induced lymphocyte apoptosis in the spleen and thymus by caspase inhibitor Q-VD-OPH. This method improved survival, reduced brain damage, and decreased susceptibility to post-stroke bacteremia in a mouse model [97].

Medications targeting aspiration have been used to decrease the risk of SAP in the chronic stages such as angiotensin-converting enzyme inhibitors which may play a theoretical role in prevention by increasing the level of substance P [21]. Cilostazol, an antiplatelet agent, may theoretically improve the production of substance $\mathrm{P}$ and dopamine by improving local circulation [98]. The clinical benefit of both medications was seen among chronic Asian and Japanese stroke patients, respectively, but not in Caucasian patients [98-100]. These medications are not likely to help in the acute stages of SAP because of the length of time needed to show the benefit.

\section{Treatment of SAP}

SAP treatment must be initiated quickly since SAP may be associated with mortality and worsening of neurological outcome [1]. SAP treatment may follow the guidelines of early hospital-acquired pneumonia therapy [101] since it mainly happens in the first few days after hospitalization [93]. The decision of the empiric antibiotic treatment depends on the individual's risk factors, disease severity, time of onset, general microbiology of SAP and the local microbiologic data of the institution [101].

\section{Conclusions}

Despite the major advances that have been achieved in understanding the pathophysiology of SAP in the last few years, there are still a large number of unanswered questions. The true incidence and outcome of SAP, especially in the critical care units, are yet to be determined. The full extent of the stroke-induced immunodepression and its clinical implications are still unknown. Many controversies in the prophylaxis against SAP are present and some clinical trials are on their way. Thus, future research should focus on elucidating the different pathophysiological mechanisms of the stroke-related immunodepression and prospective clinical studies are required to answer the uncertainty of SAP outcomes, incidence and prophylaxis. On the way to achieve that, different heterogeneity factors across the clinical studies must be overcome. Clinical studies need to develop a unified diagnosis of SAP, strict 
inclusion criteria for stroke patients and differentiation among different stroke subtypes, and they need to have long-term follow-ups with a clear definition of functional outcome, especially among critical care studies. Experimental research should elucidate the rest of the unknown interactions between the brain and immune system which may lead to the discovery of new experimental treatments that may have clinical implications. All of that may lead to developing new treatment and prophylactic measures that aid in limiting this complication which may have detrimental effects on stroke outcomes.

\section{Disclosure Statement}

The authors report no conflicts of interest or disclosures.

\section{References}

1 Vermeij FH, Scholte op Reimer WJ, de Man $\mathrm{P}$, et al: Stroke-associated infection is an independent risk factor for poor outcome after acute ischemic stroke: data from the Netherlands Stroke Survey. Cerebrovasc Dis 2009; 27:465-471.

2 Roger VL, Go AS, Lloyd-Jones DM, et al: Heart disease and stroke statistics - 2012 update: a report from the American Heart Association. Circulation 2012;125:e2-e220.

3 Koennecke HC, Belz W, Berfelde D, et al: Factors influencing in-hospital mortality and morbidity in patients treated on a stroke unit. Neurology 2011;77:965-972.

4 Katzan IL, Dawson NV, Thomas CL, Votruba ME, Cebul RD: The cost of pneumonia after acute stroke. Neurology 2007;68:19381943.

5 Wilson RD: Mortality and cost of pneumonia after stroke for different risk groups. J Stroke Cerebrovasc Dis 2012;21:61-67.

6 Chamorro A, Meisel A, Planas AM, Urra X, van de Beek D, Veltkamp R: The immunology of acute stroke. Nat Rev Neurol 2012;8:401410.

7 Dirnagl U, Klehmet J, Braun JS, et al: Strokeinduced immunodepression: experimental evidence and clinical relevance. Stroke 2007; 38:770-773.

8 Bershad EM, Feen ES, Hernandez OH, Suri MF, Suarez JI: Impact of a specialized neurointensive care team on outcomes of critically ill acute ischemic stroke patients. Neurocrit Care 2008;9:287-292.

9 Kwon HM, Jeong SW, Lee SH, Yoon BW: The pneumonia score: a simple grading scale for prediction of pneumonia after acute stroke. Am J Infect Control 2006;34:64-68.

10 Teramoto S: Novel preventive and therapuetic strategy for post-stroke pneumonia. Expert Rev Neurother 2009;9:1187-1200.

11 Hilker R, Poetter C, Findeisen N, et al: Nosocomial pneumonia after acute stroke: implications for neurological intensive care medicine. Stroke 2003;34:975-981.

12 Kasuya Y, Hargett JL, Lenhardt R, et al: Ventilator-associated pneumonia in critically ill stroke patients: frequency, risk factors, and outcomes. J Crit Care 2011;26:273-279.

13 Horan TC, Andrus M, Dudeck MA: CDC/ NHSN surveillance definition of health care- associated infection and criteria for specific types of infections in the acute care setting. Am J Infect Control 2008;36:309-332.

14 Josephson SA, Moheet AM, Gropper MA, Nichols AD, Smith WS: Ventilator-associated pneumonia in a neurologic intensive care unit does not lead to increased mortality. Neurocrit Care 2010;12:155-158.

15 Walter U, Knoblich R, Steinhagen V, Donat $\mathrm{M}$, Benecke R, Kloth A: Predictors of pneumonia in acute stroke patients admitted to a neurological intensive care unit. J Neurol 2007; 254:1323-1329.

16 Huang JY, Zhang DY, Yao Y, Xia QX, Fan QQ: Training in swallowing prevents aspiration pneumonia in stroke patients with dysphagia. J Int Med Res 2006;34:303-306.

17 Lim SH, Lieu PK, Phua SY, et al: Accuracy of bedside clinical methods compared with fiberoptic endoscopic examination of swallowing (FEES) in determining the risk of aspiration in acute stroke patients. Dysphagia 2001; 16:1-6.

18 Lakshminarayan $\mathrm{K}$, Tsai AW, Tong X, et al: Utility of dysphagia screening results in predicting poststroke pneumonia. Stroke 2010; 41:2849-2854.

19 Jia YX, Sekizawa K, Ohrui T, Nakayama K, Sasaki H: Dopamine $\mathrm{D}_{1}$ receptor antagonist inhibits swallowing reflex in guinea pigs. Am J Physiol 1998;274:R76-R80.

20 Nakagawa T, Ohrui T, Sekizawa K, Sasaki H: Sputum substance $\mathrm{P}$ in aspiration pneumonia. Lancet 1995;345:1447.

21 Arai T, Yoshimi N, Fujiwara H, Sekizawa K Serum substance $\mathrm{P}$ concentrations and silent aspiration in elderly patients with stroke. Neurology 2003;61:1625-1626.

22 Chastre J, Fagon JY: Ventilator-associated pneumonia. Am J Respir Crit Care Med 2002; 165:867-903.

23 Upadya A, Thorevska N, Sena KN, Manthous C, Amoateng-Adjepong Y: Predictors and consequences of pneumonia in critically ill patients with stroke. J Crit Care 2004; 19:16-22.

24 Weimar C, Roth MP, Zillessen G, et al: Complications following acute ischemic stroke. Eur Neurol 2002;48:133-140.

25 Dziewas R, Ritter M, Schilling M, et al: Pneumonia in acute stroke patients fed by nasogas- tric tube. J Neurol Neurosurg Psychiatry 2004; 75:852-856.

26 Chamorro A, Urra X, Planas AM: Infection after acute ischemic stroke: a manifestation of brain-induced immunodepression. Stroke 2007;38:1097-1103.

27 Howard RJ, Simmons RL: Acquired immunologic deficiencies after trauma and surgical procedures. Surg Gynecol Obstet 1974;139: 771-782.

28 Meisel C, Schwab JM, Prass K, Meisel A, Dirnagl U: Central nervous system injury-induced immune deficiency syndrome. Nat Rev Neurosci 2005;6:775-786.

29 Prass K, Meisel C, Hoflich C, et al: Stroke-induced immunodeficiency promotes spontaneous bacterial infections and is mediated by sympathetic activation reversal by poststroke T helper cell type 1-like immunostimulation. J Exp Med 2003;198:725-736.

30 Prass K, Braun JS, Dirnagl U, Meisel C, Meisel A: Stroke propagates bacterial aspiration to pneumonia in a model of cerebral ischemia. Stroke 2006;37:2607-2612.

31 Haeusler KG, Schmidt WU, Fohring F, et al: Cellular immunodepression preceding infectious complications after acute ischemic stroke in humans. Cerebrovasc Dis 2008;25:50-58.

32 Liesz A, Hagmann S, Zschoche C, et al: The spectrum of systemic immune alterations after murine focal ischemia: immunodepression versus immunomodulation. Stroke 2009; 40:2849-2858.

33 Schulte-Herbruggen O, Quarcoo D, Meisel A, Meisel C: Differential affection of intestinal immune cell populations after cerebral ischemia in mice. Neuroimmunomodulation 2009; 16:213-218.

34 Vogelgesang A, Grunwald U, Langner S, et al: Analysis of lymphocyte subsets in patients with stroke and their influence on infection after stroke. Stroke 2008;39:237-241.

35 Walter U, Kolbaske S, Patejdl R, et al: Insular stroke is associated with acute sympathetic hyperactivation and immunodepression. Eur J Neurol 2013;20:153-159.

36 Marklund N, Peltonen M, Nilsson TK, Olsson T: Low and high circulating cortisol levels predict mortality and cognitive dysfunction early after stroke. J Intern Med 2004; 256:15-21. 
37 Bernik TR, Friedman SG, Ochani M, et al: Pharmacological stimulation of the cholinergic antiinflammatory pathway. J Exp Med 2002;195:781-788.

38 Tuosto L, Cundari E, Gilardini Montani MS, Piccolella E: Analysis of susceptibility of mature human $\mathrm{T}$ lymphocytes to dexamethasone-induced apoptosis. Eur J Immunol 1994; 24:1061-1065.

39 Whalen MJ, Carlos TM, Clark RS, et al: The relationship between brain temperature and neutrophil accumulation after traumatic brain injury in rats. Acta Neurochir Suppl 1997;70:260-261.

40 Dietrich WD, Chatzipanteli K, Vitarbo E, Wada K, Kinoshita K: The role of inflammatory processes in the pathophysiology and treatment of brain and spinal cord trauma. Acta Neurochir Suppl 2004;89:69-74.

41 Takagi K, Ginsberg MD, Globus MY, Martinez E, Busto R: Effect of hyperthermia on glutamate release in ischemic penumbra after middle cerebral artery occlusion in rats. Am J Physiol 1994;267:H1770-H1776.

42 Hajat C, Hajat S, Sharma P: Effects of poststroke pyrexia on stroke outcome: a metaanalysis of studies in patients. Stroke 2000;31: 410-414.

43 Wright WL: Sodium and fluid management in acute brain injury. Curr Neurol Neurosci Rep 2012;12:466-473.

44 Huang WY, Weng WC, Peng TI, et al: Association of hyponatremia in acute stroke stage with three-year mortality in patients with firstever ischemic stroke. Cerebrovasc Dis 2012; 34:55-62.

45 Martinez MA, Pena JM, Fernandez A, et al: Time course and prognostic significance of hemostatic changes in sepsis: relation to tumor necrosis factor-alpha. Crit Care Med 1999;27: 1303-1308.

46 Millns B, Gosney M, Jack CI, Martin MV, Wright AE: Acute stroke predisposes to oral gram-negative bacilli - A cause of aspiration pneumonia? Gerontology 2003;49: 173-176.

47 Gosney MA, Martin MV, Wright AE, Gallagher M: Enterobacter sakazakii in the mouths of stroke patients and its association with aspiration pneumonia. Eur J Intern Med 2006; 17:185-188.

48 Hassan A, Khealani BA, Shafqat S, et al: Stroke-associated pneumonia: microbiological data and outcome. Singapore Med J 2006; 47:204-207.

49 Kostadima E, Kaditis AG, Alexopoulos EI, Zakynthinos E, Sfyras D: Early gastrostomy reduces the rate of ventilator-associated pneumonia in stroke or head injury patients. Eur Respir J 2005;26:106-111.

50 Yilmaz GR, Cevik MA, Erdinc FS, Ucler S, Tulek N: The risk factors for infections acquired by cerebral hemorrhage and cerebral infarct patients in a neurology intensive care unit in Turkey. Jpn J Infect Dis 2007;60:8791.
51 Dettenkofer M, Ebner W, Els T, et al: Surveillance of nosocomial infections in a neurology intensive care unit. J Neurol 2001;248:959964.

52 Westendorp WF, Nederkoorn PJ, Vermeij JD, Dijkgraaf MG, van de Beek D: Post-stroke infection: a systematic review and meta-analysis. BMC Neurol 2011;11:110.

53 Sui R, Zhang L: Risk factors of stroke-associated pneumonia in Chinese patients. Neurol Res 2011;33:508-513.

54 Yeh SJ, Huang KY, Wang TG, et al: Dysphagia screening decreases pneumonia in acute stroke patients admitted to the stroke intensive care unit. J Neurol Sci 2011;306:38-41.

55 Commichau C, Scarmeas N, Mayer SA: Risk factors for fever in the neurologic intensive care unit. Neurology 2003;60:837-841.

56 Yan F, Zhang D, Xu H, Guo H: Risk factors for fever in critically ill patients with acute newonset stroke. Neurol Res 2008;30:394-399.

57 Hassan AE, Chaudhry SA, Zacharatos H, et al: Increased rate of aspiration pneumonia and poor discharge outcome among acute ischemic stroke patients following intubation for endovascular treatment. Neurocrit Care 2012; 16:246-250.

58 Yang CC, Shih NC, Chang WC, Huang SK, Chien CW: Long-term medical utilization following ventilator-associated pneumonia in acute stroke and traumatic brain injury patients: a case-control study. BMC Health Serv Res 2011;11:289.

59 Broadley S, Croser D, Cottrell J, et al: Predictors of prolonged dysphagia following acute stroke. J Clin Neurosci 2003;10:300-305.

60 Dziedzic T, Pera J, Klimkowicz A, et al: Serum albumin level and nosocomial pneumonia in stroke patients. Eur J Neurol 2006;13:299-301.

61 Dziedzic T, Slowik A, Pera J, Szczudlik A: Association between hyperglycemia, heart failure and mortality in stroke patients. Eur J Neurol 2009;16:251-256.

62 Gosney M, Martin MV, Wright AE: The role of selective decontamination of the digestive tract in acute stroke. Age Ageing 2006;35: 42-47.

63 Ickenstein GW, Riecker A, Hohlig C, et al: Pneumonia and in-hospital mortality in the context of neurogenic oropharyngeal dysphagia (NOD) in stroke and a new NOD stepwise concept. J Neurol 2010;257:1492-1499.

64 Ifejika-Jones NL, Arun N, Peng H, Elizabeth A, Grotta JC, Francisco GE: The interaction of aspiration pneumonia with demographic and cerebrovascular disease risk factors is predictive of discharge level of care in acute stroke patient. Am J Phys Med Rehabil 2012; 91:141-147.

65 Ingeman A, Andersen G, Hundborg HH, Svendsen ML, Johnsen SP: Processes of care and medical complications in patients with stroke. Stroke 2011;42:167-172.

66 Minnerup J, Wersching H, Brokinkel B, et al: The impact of lesion location and lesion size on poststroke infection frequency. J Neurol Neurosurg Psychiatry 2010;81:198-202.
67 Saposnik G, Black SE, Hakim A, et al: Age disparities in stroke quality of care and delivery of health services. Stroke 2009;40:3328-3335.

68 Aslanyan S, Weir CJ, Diener HC, Kaste M, Lees KR: Pneumonia and urinary tract infection after acute ischaemic stroke: a tertiary analysis of the GAIN International trial. Eur J Neurol 2004;11:49-53.

69 Chen CM, Hsu HC, Tsai WS, Chang CH, Chen $\mathrm{KH}$, Hong CZ: Infections in acute older stroke inpatients undergoing rehabilitation. Am J Phys Med Rehabil 2012;91:211-219.

70 Chumbler NR, Williams LS, Wells CK, et al: Derivation and validation of a clinical system for predicting pneumonia in acute stroke. Neuroepidemiology 2010;34:193-199.

71 Cuesy PG, Sotomayor PL, Pina JO: Reduction in the incidence of poststroke nosocomial pneumonia by using the 'turn-mob' program. J Stroke Cerebrovasc Dis 2010;19:23-28.

72 Field TS, Green TL, Roy K, Pedersen J, Hill MD: Trends in hospital admission for stroke in Calgary. Can J Neurol Sci 2004;31:387-393.

73 Finlayson O, Kapral M, Hall R, et al: Risk factors, inpatient care, and outcomes of pneumonia after ischemic stroke. Neurology 2011; 77:1338-1345.

74 Grau AJ, Buggle F, Schnitzler P, Spiel M, Lichy C, Hacke W: Fever and infection early after ischemic stroke. J Neurol Sci 1999;171: 115-120.

75 Hamidon BB, Raymond AA, Norlinah MI, Jefferelli SB: The predictors of early infection after an acute ischaemic stroke. Singapore Med J 2003;44:344-346.

76 Hinchey JA, Shephard T, Furie K, Smith D, Wang D, Tonn S: Formal dysphagia screening protocols prevent pneumonia. Stroke 2005; 36:1972-1976.

77 Hong KS, Kang DW, Koo JS, et al: Impact of neurological and medical complications on 3-month outcomes in acute ischaemic stroke. Eur J Neurol 2008;15:1324-1331.

78 Johnston KC, Li JY, Lyden PD, et al: Medical and neurological complications of ischemic stroke: experience from the RANTTAS trial. RANTTAS Investigators. Stroke 1998;29: 447-453.

79 Jones EM, Albright KC, Fossati-Bellani M, Siegler JE, Martin-Schild S: Emergency department shift change is associated with pneumonia in patients with acute ischemic stroke. Stroke 2011;42:3226-3230.

80 Katzan IL, Cebul RD, Husak SH, Dawson NV, Baker DW: The effect of pneumonia on mortality among patients hospitalized for acute stroke. Neurology 2003;60:620-625.

81 Ota K, Saitoh E, Baba M, Sonoda S: The secretion severity rating scale: a potentially useful tool for management of acute-phase fasting stroke patients. J Stroke Cerebrovasc Dis 2011;20:183-187.

82 Sellars C, Bowie L, Bagg J, et al: Risk factors for chest infection in acute stroke: a prospective cohort study. Stroke 2007;38:2284-2291. 
83 Hanchaiphiboolkul S: Risk factors for early infection after an acute cerebral infarction. J Med Assoc of Thai 2005;88:150-155.

84 Kolb G, Broker M: State of the art in aspiration assessment and the idea of a new non invasive predictive test for the risk of aspiration in stroke. J Nutrition Health Aging 2009;13: 429-433.

85 Falsetti P, Acciai C, Palilla R, et al: Oropharyngeal dysphagia after stroke: incidence, diagnosis, and clinical predictors in patients admitted to a neurorehabilitation unit. J Stroke Cerebrovasc Dis 2009;18:329-335.

86 Lipson DM, Sangha H, Foley NC, Bhogal S, Pohani G, Teasell RW: Recovery from stroke: differences between subtypes. Int J Rehabil Res 2005;28:303-308.

87 Teasell R, Foley N, Doherty T, Finestone H: Clinical characteristics of patients with brainstem strokes admitted to a rehabilitation unit. Arch Phys Med Rehabil 2002;83: 1013-1016.

88 Seo WK, Yu SW, Kim JH, Park KW, Koh SB: The impact of hyperthermia and infection on acute ischemic stroke patients in the intensive care unit. Neurocrit Care 2008;9:183-188.

89 Adams HP Jr, del Zoppo G, Alberts MJ, et al: Guidelines for the early management of adults with ischemic stroke: a guideline from the American Heart Association/American Stroke Association Stroke Council, Clinical Cardiology Council, Cardiovascular Radiol- ogy and Intervention Council, and the Atherosclerotic Peripheral Vascular Disease and Quality of Care Outcomes in Research Interdisciplinary Working Groups: The American Academy of Neurology affirms the value of this guideline as an educational tool for neurologists. Circulation 2007;115:e478-e534.

90 Gomes CA Jr, Lustosa SA, Matos D, Andriolo RB, Waisberg DR, Waisberg J: Percutaneous endoscopic gastrostomy versus nasogastric tube feeding for adults with swallowing disturbances. Cochrane Database Syst Rev 2012; 3:CD008096.

91 Meisel C, Prass K, Braun J, et al: Preventive antibacterial treatment improves the general medical and neurological outcome in a mouse model of stroke. Stroke 2004;35:2-6.

92 Harms H, Prass K, Meisel C, et al: Preventive antibacterial therapy in acute ischemic stroke: a randomized controlled trial. PLoS One 2008;3:e2158.

93 Chamorro A, Horcajada JP, Obach V, et al: The Early Systemic Prophylaxis of Infection After Stroke study: a randomized clinical trial. Stroke 2005;36:1495-1500.

94 Schwarz S, Al-Shajlawi F, Sick C, Meairs S, Hennerici MG: Effects of prophylactic antibiotic therapy with mezlocillin plus sulbactam on the incidence and height of fever after severe acute ischemic stroke: the Mannheim infection in stroke study (MISS). Stroke 2008; 39:1220-1227.
95 Westendorp WF, Vermeij JD, Vermeij F, et al: Antibiotic therapy for preventing infections in patients with acute stroke. Cochrane Database Syst Rev 2012;1:CD008530.

96 Nederkoorn PJ, Westendorp WF, Hooijenga IJ, et al: Preventive antibiotics in stroke study: rationale and protocol for a randomised trial. Int J Stroke 2011;6:159-163.

97 Braun JS, Prass K, Dirnagl U, Meisel A, Meisel C: Protection from brain damage and bacterial infection in murine stroke by the novel caspase-inhibitor Q-VD-OPH. Exp Neurol 2007;206:183-191.

98 Shinohara Y: Antiplatelet cilostazol is effective in the prevention of pneumonia in ischemic stroke patients in the chronic stage. Cerebrovasc Dis 2006;22:57-60.

99 Ohkubo T, Chapman N, Neal B, et al: Effects of an angiotensin-converting enzyme inhibitor-based regimen on pneumonia risk. Am J Respir Crit Care Med 2004;169: 1041-1045.

100 Shinohara Y, Origasa H: Post-stroke pneumonia prevention by angiotensin-converting enzyme inhibitors: results of a meta-analysis of five studies in Asians. Adv Ther 2012;29: 900-912.

101 Torres A, Ferrer M, Badia JR: Treatment guidelines and outcomes of hospital-acquired and ventilator-associated pneumonia. Clin Infect Dis 2010;51(suppl 1):S48S53. 